\title{
Airfoil Analysis with Uncertain Geometry using the Probabilistic Collocation method
}

\author{
G.J.A. Loeven*† and H. Bijl ${ }^{\ddagger}$ \\ Delft University of Technology, The Netherlands
}

\begin{abstract}
Due to manufacturing tolerances, the airfoil of a wing after production is never exactly the same as the designed airfoil. Also during operation the geometry may change due to aerodynamic loading, icing or wear of the construction. The geometry can, therefore, be treated as uncertain. Uncertainties in the geometry of an airfoil are expected to have a significant influence on quantities like lift and drag. Computational Fluid Dynamics is a tool to investigate the flow around an airfoil, which is characterized by large time consuming computations. Since uncertainty quantification increases the amount of computational work, efficiency is of great importance. To limit the number of uncertain parameters, the geometry is parameterized using a few parameters. The geometric uncertainties are then treated as parametric uncertainties, which are efficiently propagated using the Probabilistic Collocation method. Results are shown for uncertain NACA 4-digit series airfoils, where the maximum camber, maximum camber position and the thickness of the airfoil are assumed to be uncertain. It is shown that geometric uncertainties have significant influence on the drag polar. The uncertainties are propagated through the system separately to see the effect of the parameter on the solution and simultaneously to investigate combined effects.
\end{abstract}

\section{Introduction}

Increasing computing power and advanced algorithms enable accurate computations of complex problems. Due to inherent physical variations the resulting computational prediction can still differ significantly from operational performance. One can think for example of variations in the freestream velocity, angle of incidence or the actual shape of the model. To include these physical variations and study their influence on the performance uncertainty quantification can be used.

Geometric uncertainties are present in reality, due to production tolerances or unknown deformations during operation. Therefore, they are of great interest, especially for industry. The geometry of a welldesigned airfoil might be a bit different when the product is made. Furthermore, the during operation the construction may deform under loading or change geometry due to for example icing. In this study the geometric uncertainty is written as a parametric uncertainty by parameterizing the geometry and assuming an uncertainty on the parameters. Examples of the shape parameters of an airfoil are the relative thickness, maximum camber, or leading edge radius, depending on the parameterization.

The effect of geometric uncertainties on CFD has been investigated before using sensitivity analysis ${ }^{1,2}$ and moment methods. ${ }^{3}$ Ilinca, Hay, and Pelletier ${ }^{1}$ treat shape sensitivities of unsteady laminar flow around a cylinder in ground proximity. Etienne, Hay, Garon, and Pelletier ${ }^{2}$ investigated shape sensitivities of flexible plates in a flow domain. Gumber, Newman, and $\mathrm{Hou}^{3}$ included first order moments in robust design optimization of a 3D flexible wing with uncertain wing geometry.

Instead of sensitivities or lower order approximations of the mean and variance, in this study the probability distribution function of the output of interest is computed. From the probability distribution more accurate intervals can be extracted, which contain for example $95 \%$ of all possible values that can occur. Since CFD is computationally intensive an efficient approach is required. This study utilizes the Polynomial Chaos framework. ${ }^{4}$ The Polynomial Chaos expansion is computed using the Probabilistic Collocation

\footnotetext{
*Corresponding author, email: G.J.A.Loeven@TUDelft.nl

$\dagger^{\dagger}$ Ph.D. researcher, Department of Aerospace Engineering, P.O. Box 5058, 2600 GB Delft, The Netherlands, member AIAA

$\ddagger$ Full Professor, Department of Aerospace Engineering, P.O. Box 5058, 2600 GB Delft, The Netherlands, member AIAA.
} 
method. ${ }^{5-7}$ Exponential convergence with respect to the polynomial order is obtained for sufficiently smooth responses and the method is non-intrusive. A non-intrusive uncertainty quantification method will enable the user to apply the method to their own commercial code, since the CFD code is treated as a black-box.

The first test case is a 4-digit series NACA airfoil. Here three parameters describe the geometry, namely the maximum camber, maximum camber location and the thickness, all in percents of the chord. Each shape parameter is assumed to be uncertain separately to investigate the effect of the parameter on the solution. After that all parameters are considered uncertain simultaneously to study the combined effect.

Secondly, flow around a NACA0012 airfoil is considered with uncertain camber and relative thickness. A commercial CFD solver is used to perform several deterministic solves to obtain a Probabilistic Collocation approximation. Computations at subsonic flow $(M=0.3)$ and at transonic flow $(M=0.8)$ are accomplished. The pressure distribution on the surface of the airfoil with uncertainty bars is shown, as well as the probability distribution functions of the lift, drag and for the transonic case the shock location.

A problem in this approach is the determination of the probability density functions of the parameters. Here truncated normal distributions are used, since this seems a reasonable assumption for produced items. The mean has the highest probability and products outside the tolerance interval are rejected.

The paper is organized as follows: first the Probabilistic Collocation method is explained after which it is demonstrated for an uncertain airfoil shape of the NACA 4-digit series using a panel method. After that flow around a NACA0012 airfoil with uncertain camber and relative thickness using a commercial CFD solver is shown. Finally, preliminary conclusions are drawn and the upcoming work for the final paper will be discussed.

\section{The Probabilistic Collocation Method}

The parameterized geometric uncertainties are propagated using the Probabilistic Collocation method, ${ }^{5-7}$ which is a non-intrusive Polynomial Chaos method. ${ }^{4}$ The Probabilistic Collocation method converges exponentially for arbitrary probability distributions with respect to the Polynomial Chaos order and requires several deterministic solves to reconstruct the Polynomial Chaos solution.

In the Probabilistic Collocation method a polynomial chaos expansion is constructed based on Lagrange polynomials. Gauss quadrature weighted by the probability density function of the uncertain parameter is used to compute the Galerkin projection and the integration of the approximation of the distribution function. By using Gauss quadrature a decoupled set of equations and a higher order approximation of the mean and variance are obtained.

\section{Probabilistic Collocation expansion}

The solution and each variable depending on the uncertain input parameter is expanded as follows:

$$
u(\boldsymbol{x}, t, \omega)=\sum_{i=1}^{N_{\mathrm{p}}} u_{i}(\boldsymbol{x}, t) h_{i}(\xi(\omega)),
$$

where the solution $u(\boldsymbol{x}, t, \omega)$, a function of the time $t$ and the random event $\omega \in \Omega$, and the number of collocation points $N_{\mathrm{p}}$. The complete probability space is given by $(\Omega, \mathcal{F}, P)$, with $\Omega$ the set of outcomes, $\mathcal{F} \subset 2^{\Omega}$ the $\sigma$-algebra of events and $P: \mathcal{F} \rightarrow[0,1]$ a probability measure. Furthermore, $u_{i}(\boldsymbol{x}, t)$ is the solution $u(\boldsymbol{x}, t, \omega)$ at the collocation point $\omega_{i} ; h_{i}$ is the Lagrange interpolating polynomial chaos corresponding to the collocation point $\omega_{i} ; \xi$ is the random basis. The Lagrange interpolation results in polynomial chaoses instead of ordinary polynomials. The Lagrange interpolating polynomial is a function in terms of the random variable $\xi(\omega)$, which is chosen on the standard domains $[-1,1],[0, \infty)$ or $(-\infty, \infty)$, such that the uncertain input parameter is a linear transformation of $\xi(\omega)$. The Lagrange interpolating polynomial chaos is the polynomial chaos $h_{i}(\xi(\omega))$ of order $N_{\mathrm{p}}-1$ that passes through the $N_{\mathrm{p}}$ collocation points, with $h_{i}\left(\xi\left(\omega_{j}\right)\right)=\delta_{i j}$. The collocation points are chosen such that they correspond to the Gauss quadrature points used to integrate the function $u(\boldsymbol{x}, t, \omega)$ in the $\omega$ domain. For convenience of notation the argument $\omega$ is omitted from here on. The solution has to be integrated in order to obtain for instance the mean or variance. To find the suitable Gauss quadrature points and weights the procedure below is followed. 
A powerful method to compute Gaussian quadrature rules is by means of the Golub-Welsch algorithm. ${ }^{8}$ This algorithm requires the recurrence coefficients ${ }^{9}$ of polynomials which are orthogonal with respect to the weighting function $w(\xi)$ of the integration. Exponential convergence for arbitrary probability distributions is obtained when the polynomials are orthogonal with respect to the probability density function of $\xi$, so $w(\xi)=f_{\xi}(\xi)$. The recurrence coefficients are computed using the discredited Stieltjes procedure, ${ }^{10}$ which is a stable method for arbitrary distribution functions.

First the recurrence coefficients are computed. Orthogonal polynomials with respect to the weighting function of the element are constructed in each element using the recurrence relation

$$
\begin{aligned}
& \Psi_{0}(\xi)=0, \Psi_{1}(\xi)=1, \\
& \Psi_{i+1}(\xi)=\left(\xi-\alpha_{i}\right) \Psi_{i}(\xi)-\beta_{i} \Psi_{i-1}, \quad i=2,3, \ldots, N_{\mathrm{p}} .
\end{aligned}
$$

Now $\alpha_{i}$ and $\beta_{i}$ are the recurrence coefficients determined by the weighting function $w(\xi)$ and $\left\{\Psi_{i}(\xi)\right\}_{i=1}^{N_{\mathrm{p}}}$ is a set of (monic) orthogonal polynomials with $\Psi_{i}(\xi)=\xi^{i}+\mathcal{O}\left(\xi^{i-1}\right), i=1,2, \ldots, N_{\mathrm{p}}$. Gander and Karp ${ }^{10}$ showed that discretizing the weighting function leads to a stable algorithm. Therefore, the discretized Stieltjes procedure is used to obtain the recurrence coefficients. From the recurrence coefficients $\alpha_{i}$ and $\beta_{i}$, $i=1, \ldots, N_{\mathrm{p}}$, the collocation points $\xi_{i}$ and corresponding weights $w_{i}$ are computed using the Golub-Welsch algorithm. ${ }^{8}$ Now the collocation points $\xi_{i}$ in the $\xi$-domain are known. They are mapped to the $\omega$-domain using the distribution function of $\xi$. The collocation points $\omega_{i}$ are then found by

$$
\omega_{i}=F_{\xi}\left(\xi_{i}\right), \quad i=1, \ldots, N_{\mathrm{p}} .
$$

For a uniformly distributed parameter and $\xi(\omega)=U(-1,1)$ the Probabilistic Collocation method results in the collocation method of Mathelin and Hussaini. ${ }^{11}$

\section{Application to a general model}

In this section the application of Probabilistic Collocation method to a general model is shown. The model is a black box and can be for example a CFD solver. It is demonstrated how the method is used when the parameter of interest is a functional of the solution, like the lift of an airfoil. Expansion (1) is substituted into the model, represented here by the operator $\mathcal{L}$, which depends on an uncertain input parameter $a(\omega)$ :

$$
\mathcal{L}(a(\omega)) u(\boldsymbol{x}, t, \omega)=S(\boldsymbol{x}, t) .
$$

A Galerkin projection on each basis $\left\{h_{i}(\xi(\omega))\right\}$ is applied:

$$
\left\langle\mathcal{L}(a(\omega)) \sum_{i=1}^{N_{\mathrm{p}}} u_{i}(\boldsymbol{x}, t) h_{i}, h_{i}\right\rangle=\left\langle S, h_{i}\right\rangle, \quad k=1, \ldots, N_{\mathrm{p}} .
$$

This projection is approximated using Gaussian quadrature, with collocation points and corresponding weights based on the input distribution. The result is a deterministic system of equations similar to equation (4) which is fully decoupled

$$
\mathcal{L}\left(a\left(\omega_{k}\right)\right) u_{k}(\boldsymbol{x}, t)=S(\boldsymbol{x}, t), \quad k=1, \ldots, N_{\mathrm{p}}
$$

The distribution function is obtained from equation (1). The mean and variance of the solution are found by

$$
\begin{aligned}
\mu_{u} & =\sum_{i=1}^{N_{\mathrm{p}}} u_{i}(\boldsymbol{x}, t) w_{i}, \\
\sigma_{u}^{2} & =\sum_{i=1}^{N_{\mathrm{p}}}\left(u_{i}(\boldsymbol{x}, t)\right)^{2} w_{i}-\left(\sum_{i=1}^{N_{\mathrm{p}}} u_{i}(\boldsymbol{x}, t) w_{i}\right)^{2},
\end{aligned}
$$

where $w_{i}$ are the weights corresponding to the collocation points $\omega_{i}$. These relations are derived from the definition of the mean and variance. 
Often the output of interest is a functional of the solution, like the lift force of an airfoil. The flow around the airfoil is computed and integration of the pressure on the surface of the airfoil yields the lift. Due to the uncertainty present in the system, the lift becomes a random variable as well. The lift coefficient is written as a polynomial chaos expansion as follows

$$
C_{l}=\sum_{i=1}^{N_{p}} C_{l i} h_{i}(\xi)
$$

where $C_{l i}$ is the lift coefficient for collocation point $\omega_{i} . C_{l i}$ follows from the deterministic computations for every collocation point.

For the Galerkin Polynomial Chaos method the functionals have to be applied to the reconstructed solution $u(\boldsymbol{x}, t, \omega)$, which can give troubles. ${ }^{12}$ If multiple uncertain parameters are present, the collocation points are found using tensor products of one dimensional points or using a sparse grid approach. ${ }^{13}$

A stochastic computation is now performed as follows:

Determine uncertain parameters

probability density functions

Compute collocation points and weights

parameter values for each collocation point

Run deterministic solver for each collocation point

deterministic results

Compute the probability distribution, mean and variance of the solution

\section{Uncertain NACA 4-digit series airfoil geometry}

The geometry of an airfoil of the NACA 4-digit series is described by three parameters. The general form is NACA $X Y Z$, where $X Y Z$ represent the following shape parameters: $X$ is the maximum camber, $Y$ the maximum camber location and $Z$ the thickness of the airfoil, all in percents of the chord. For example NACA5412 (Figure 1) is an airfoil with 5 percent maximum camber, located at 40 percent of the chord and the thickness is 12 percent of the chord.

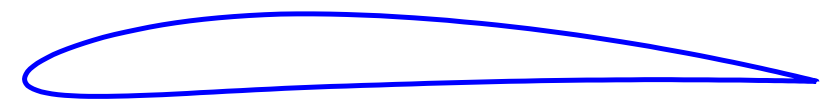

Figure 1. NACA5412 airfoil

The next sections show the effect of uncertainties on all three shape parameters separately and simultaneously. These uncertainties occur in reality when due to the production process the final product differs from the design. Three things have to be taken into account when this approach is followed, first the choice of input distribution of a shape parameter is not obvious. Here truncated normal distributions are used, since this seems a reasonable assumption for products. The mean has the highest probability and products outside the tolerance interval are rejected. The coefficient of variation is set to $C V=0.1$. Secondly, when multiple uncertain shape parameters are considered, they are not likely to be independent. All parameters together determine the actual shape, so an uncertain shape will affect all parameters. And thirdly, there are different ways of parameterizing the shape of an airfoil. The effect of the way of parameterization is not investigated here.

The results in Figure 2 show the $C_{l^{-}} \alpha$ graph for a range of angles of attack of -1 to 15 degrees. The lift coefficient is obtained using the panel method with stall and transition models, ${ }^{14,15}$ implemented in JavaFOIL. ${ }^{16}$ Here the Eppler stall model combined with the $e^{N}$ transition model ${ }^{17}$ are used. For this range 
of angles of attack the used panel method accurately predicts the lift and drag of the airfoil. The flow around the airfoil is set to a Reynolds number of $3 \cdot 10^{6}$ at sea level. The uncertainty bars show the interval on the graph that contains $95 \%$ of all possible values based on the input uncertainty. The bars are obtained from the probability distribution of the lift coefficient, which is obtained using the Probabilistic Collocation method. A second order approximation is used, meaning 3 deterministic solves for each parameter. The accuracy is checked by computing a fourth order approximation with 5 solves, which showed no significant improvement. Figure 2 shows that each parameter separately has a different effect on the lift coefficient.

\section{Uncertain maximum camber: NACAX412}

The results for the uncertain maximum camber $X$ are shown in Figure 2(a). The mean is set to $\mu_{X}=5 \%$ of the chord and the coefficient of variation to $C V_{X}=0.1$. A truncated normal distribution is used for $X$, the distribution is truncated to [3.5\%,6.5\%]. However, $60 \%$ of the possible values for $X$ are inside the interval $[4.58 \%, 5.42 \%]$. From Figure $2(\mathrm{a})$ it can be seen that the uncertain camber shifts the $C_{l^{-}} \alpha$ graph up and down. The coefficient of variation of the lift coefficient decreases when the angle of attack is increased, see Figure 3(a). The input coefficient of variation of $10 \%$ increases $15 \%$ for low angles of attack and decreased to $5 \%$ near the maximum lift coefficient.

\section{Uncertain maximum camber location: NACA5Y12}

The results in Figure 2 (b) are for a mean maximum camber location at $40 \%$ of the chord with coefficient of variation of $C V_{Y}=0.1$. The interval $[36.5 \%, 43.5 \%$ ] contains $60 \%$ of all possible values and the distribution is truncated at $[29 \%, 51 \%]$. An uncertain maximum camber location $Y$ has significant less influence on the $C_{l^{-}} \alpha$ graph compared to the maximum camber. Figure $3(\mathrm{~b})$ shows that for low angles of attack the coefficient of the variation is less than $5 \%$ compared to an input of $10 \%$. Near the maximum lift coefficient, the coefficient of variation decreases to less than $0.5 \%$. The lift coefficient is not sensitive to variations in the maximum camber location of the airfoil.

\section{Uncertain maximum thickness: NACA54Z}

Figure 2(c) shows that the uncertain thickness $Z$ does not influence the $C_{l}$ value at low angles of attack much. Again an input coefficient of variation of $C V_{Z}=0.1$ is used with a mean thickness of $12 \%$ of the chord. The thickness is truncated at [8.5\%,15.5\%], $60 \%$ falls in $[11 \%, 13 \%]$. The coefficient of variation of the lift coefficient is about $1 \%$ for low angles of attack, when separation starts moving forward from the trailing edge the coefficient increases rapidly to $6 \%$ at an angle of attack of $15^{\circ}$, as can be seen in Figure 3(c). The turbulent separation is influences mostly by the thickness. On a thinner airfoil separation starts at a lower angle of attack than for a thicker airfoil. This leads to larger uncertainty bars at higher angles of attack.

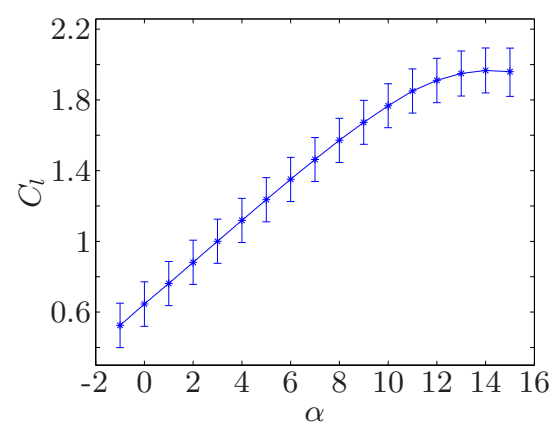

(a) Maximum camber $\mu_{X}=5 \%$

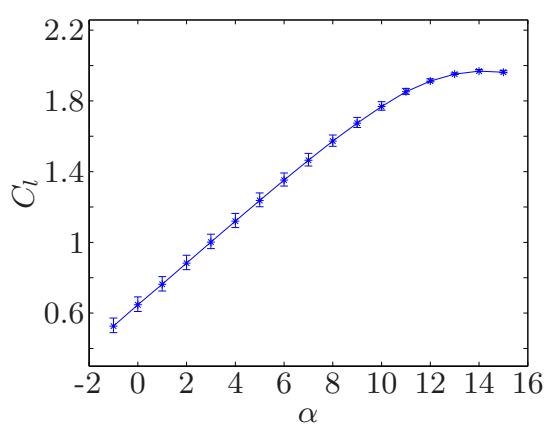

(b) Max. camber pos. $\mu_{Y}=40 \%$

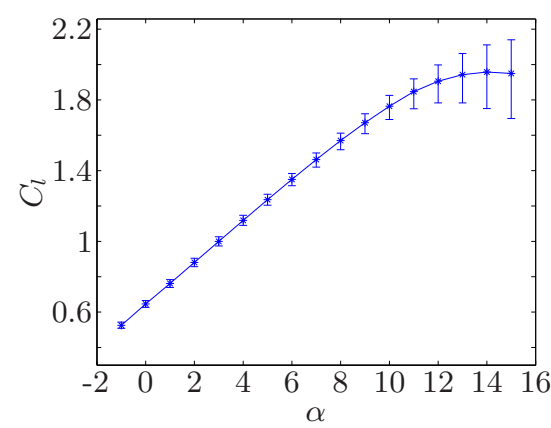

(c) Thickness $\mu_{Z}=12 \%$

Figure 2. The lift coefficient of a NACA5412 airfoil with (a) the maximum camber $X$, (b) maximum camber location $Y$ and (c) thickness $Z$ uncertain, assuming a truncated normal distribution with a coefficient of variation of $10 \%$. The uncertainty bars show $95 \%$ of all possible values bases on the probability distribution function obtained from a 2nd order Probabilistic Collocation computation (3 deterministic solves). 


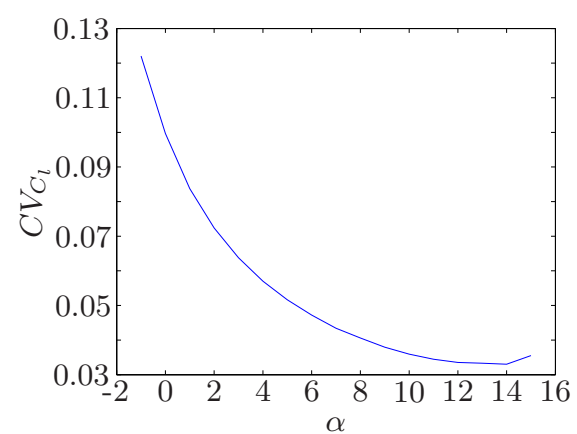

(a) Maximum camber $\mu_{X}=5 \%$

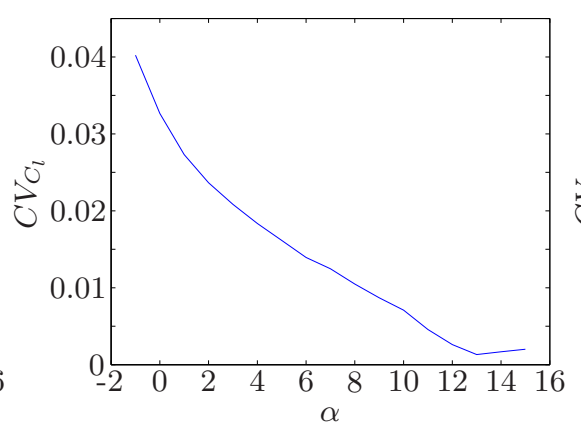

(b) Max. camber pos. $\mu_{Y}=40 \%$

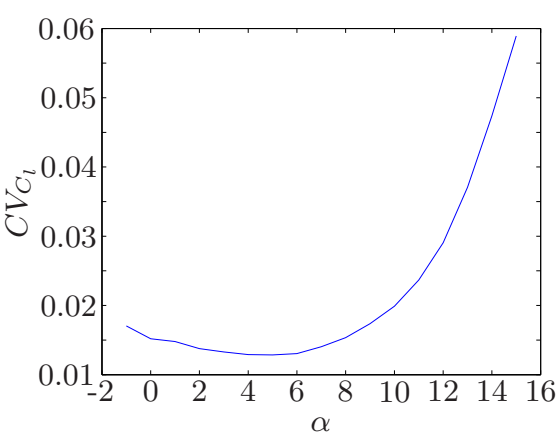

(c) Thickness $\mu_{Z}=12 \%$

Figure 3. The coefficient of variation of the lift coefficient of a NACA5412 airfoil with (a) the maximum camber $X$, (b) maximum camber location $Y$ and (c) thickness $Z$ uncertain, assuming a truncated normal distribution with a coefficient of variation of $10 \%$.

\section{Uncertain airfoil: NACAXYZ}

The previous sections showed that each parameter has a different effect on the solution. Now all parameters are assumed to be uncertain simultaneously. For example strains in the material can lead to a different maximum camber, maximum camber location and thickness. Probably the parameters are correlated in reality, however, these results assume independency.

All parameters have a truncated Gaussian distribution with a coefficient of variation of $C V_{X Y Z}=0.1$, the mean airfoil is the NACA5412 airfoil shown in Figure 1. The input uncertainty is propagated using a 2nd order probabilistic collocation approach, requiring 27 deterministic computations. Figure 4 (a) and (b) show the lift and drag coefficient with respect to the angle of attack, including uncertainty bars which indicate the interval that contains $95 \%$ of all possible values. It is shown in Figure 4(c) that the input uncertainty results in a lift coefficient with a coefficient of variation between 5 and $13 \%$ and the drag has on average a coefficient of variation of $6 \%$. The drag polar is shown in Figures 5(a) and (b). Figures 5(a) shows the 95\% uncertainty bars from Figures 4(a) and (b) in a drag polar. For the lift coefficient the bars are vertical and for the drag the uncertainty bars are horizontal. To clarify the figure, the bars are connected resulting in two areas, one originating from the drag coefficient and one from the lift coefficient. Together they form the shaded area which contains $95 \%$ of all possible values is shown in Figure 5(b). The dashed blue line shows the contribution of the drag coefficient and the red dashdot line the contribution of the lift coefficient. For low angles of attack $\left(\leq 5^{\circ}\right)$, inside the drag bucket, the uncertainty of the drag coefficient is most important. For angles of attack larger than $5^{\circ}$ the lift coefficient's uncertainty bars determine the uncertainty region.

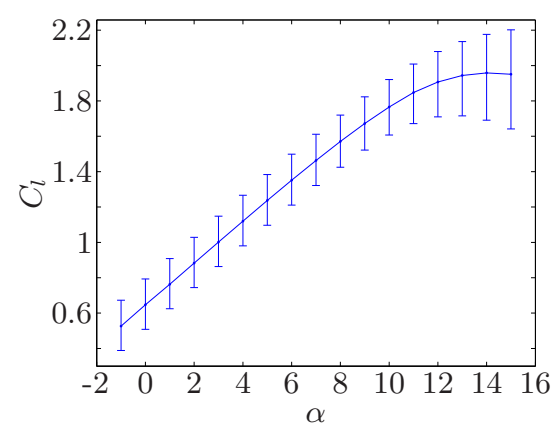

(a) $C_{l}-\alpha$

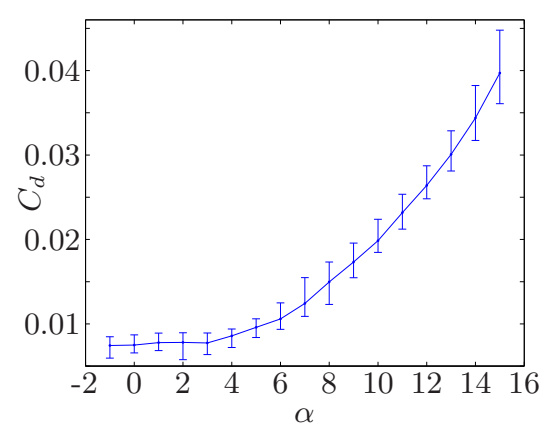

(b) $C_{d}-\alpha$

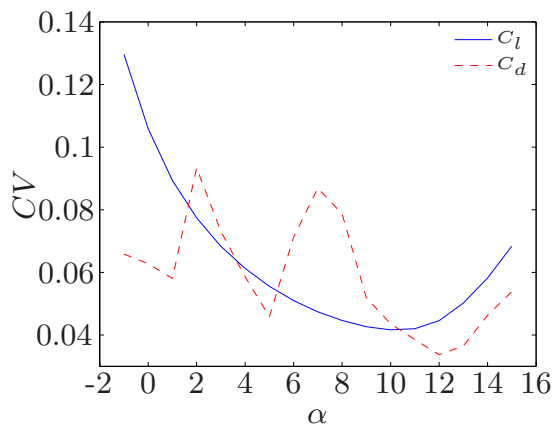

(c) Coefficients of variation

Figure 4. Characteristics of a NACA5412 airfoil with all three shape parameters truncated Gaussian distributed with a coefficient of variation of $10 \%$. The lift (a) and drag (b) coefficient with respect to the angle of attack and the coefficient of variation (c) of the lift (-) and drag coefficient (--). 

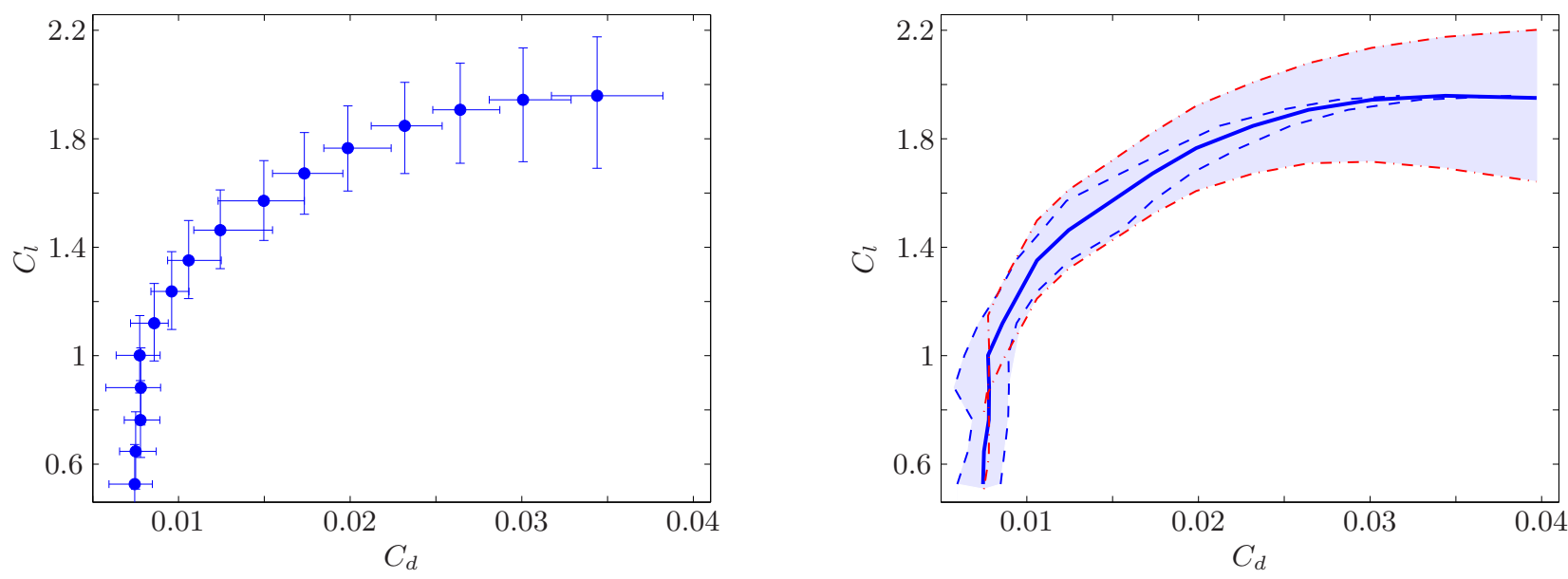

Figure 5. The mean drag polar (-) for a NACA5412 with uncertain geometry, all three parameters truncated Gaussian distributed with a coefficient of variation of $10 \%$. The uncertainty bars (a) indicate the interval which contain $95 \%$ of all possible values, which is obtained from the probability distribution function using a 2nd order Probabilistic Collocation approximation (27 deterministic solves). The bars are connected to from the shaded $95 \%$ uncertainty area. The blue dashed line (--) shows the interval from the drag coefficient and the red dashdot line (---) the interval from the lift coefficient.

\section{Uncertain NACA0012 using a commercial CFD solver}

Two cases are presented for the NACA0012 airfoil with uncertain geometry. The first case is at a Mach number of $M=0.3$. Here the flow is modelled using the Reynolds averaged Navier-Stokes equations and a turbulence model. The second case is at $M=0.8$, where the flow is solved using the Euler equations.

\section{A. Subsonic flow at $M=0.3$}

The previous section made clear that the camber and relative thickness have the largest effect on the lift and drag of an airfoil. In this section flow around a NACA0012 airfoil with uncertain camber and relative thickness is considered. The uncertainties are propagated using the Probabilistic Collocation method. The airfoil is under an angle of attack of 5 degrees and the Reynolds number is equal to 3 million. The deterministic computations are performed using the Fine ${ }^{\mathrm{TM}} / \mathrm{Hexa}$ solver by Numeca Int. on a grid of 80,000 cells. The grid layout is shown in Figure 6. The flow is modeled by the Reynolds averaged Navier-Stokes equations

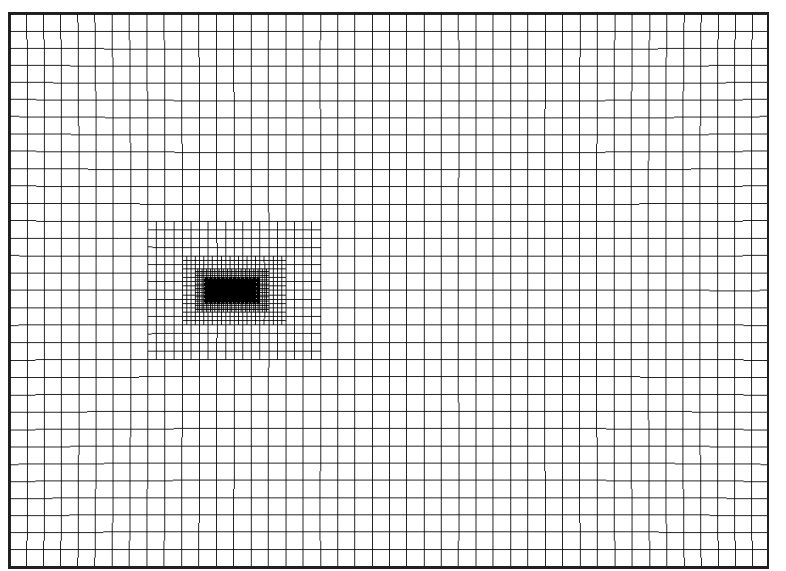

(a)

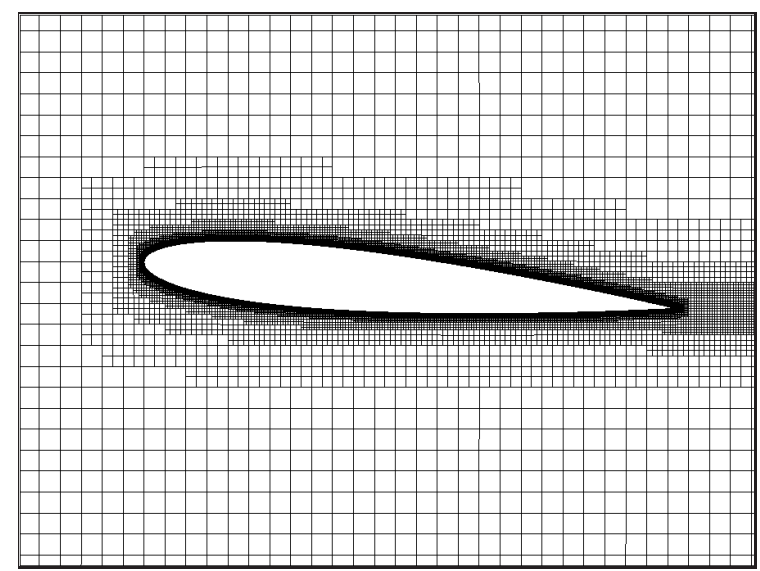

(b)

Figure 6. The computational mesh layout(a) and a detailed view of the airfoil(b). 
using the Spalart-Allmaras turbulence model. The air properties are at 0m ISA. The mean free stream Mach number is set to $M=0.3$ and the free stream flow is fully turbulent. The pressure coefficient around the mean airfoil is shown in Figure 7.

The uncertainties are the camber $c$ and the relative thickness $t$ of the airfoil, both are assumed to have a truncated normal distribution. The camber is truncated at $[-1 \%,+1 \%]$ with a standard deviation of $\sigma_{c}=$ $0.4472 \%$. The relative thickness is truncated at $[11 \%, 13 \%]$ with a standard deviation of $\sigma_{t}=0.425 \%$ Both uncertain parameters are assumed to be independent and propagated through the system simultaneously. A second order Probabilistic Collocation approximation is used, which requires 9 deterministic solves.

Figure 8 shows the pressure coefficient at the surface of the airfoil. The mean is shown by the solid line, whereas the uncertainty bars indicate the $95 \%$ or $2 \sigma$ interval. The uncertainties in camber and relative thickness have a significant influence on the pressure near the leading edge and between 20 and 60 percent of the chord.

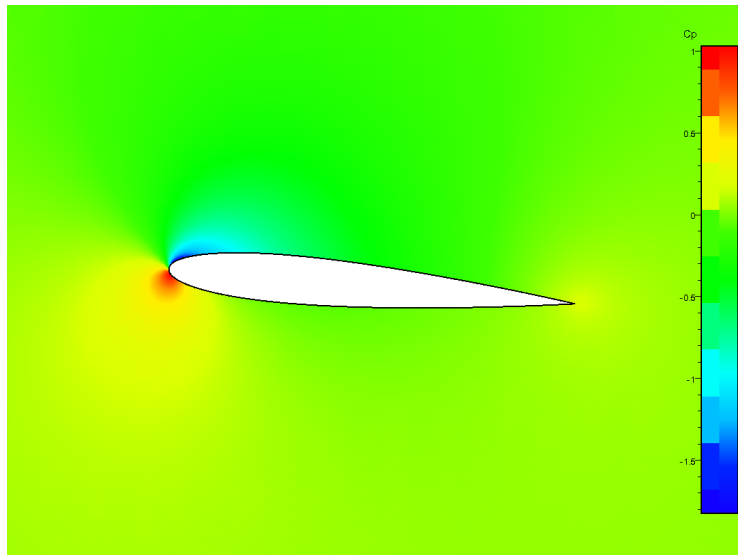

Figure 7. Pressure coefficient around the mean airfoil at $M=0.3$.

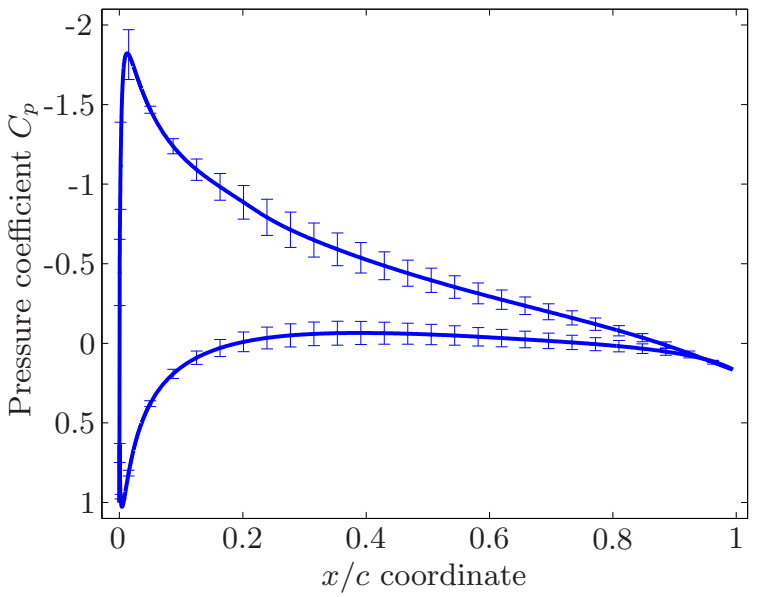

Figure 8. Pressure distribution at the surface of the airfoil. The mean is indicated with the solid line $(-)$, the uncertainty bars show the $2 \sigma$ region (containing $95 \%$ of all values).

The probability distribution functions of the lift and drag coefficient are shown in figure 9 (a) and (b). The deterministic values are $C_{l}=0.5416$ and $C_{d}=0.0127$. From the uncertainties in the camber and relative thickness the mean values result in $\mu_{C_{l}}=0.5405$ and $\mu_{C_{d}}=0.0128$, which are very close to the deterministic values. The standard deviations are $\sigma_{C_{l}}=0.0479$ and $\sigma_{C_{d}}=0.0002$, resulting in coefficients of variation of $C V_{C_{l}}=8.86 \%$ and $C V_{C_{d}}=1.8 \%$. The variation in the lift coefficient is much higher than the variation in drag coefficient based on the present uncertainties. Both variations are significant, since the $95 \%$ uncertainty interval $(2 \sigma)$ of the lift coefficient covers 190 liftcounts and of the drag coefficient it covers 80 dragcounts.

\section{B. Transonic flow at $M=0.8$}

The airfoil is under an angle of attack of 5 degrees and again the relative thickness and camber are assumed to be uncertain. The deterministic computations are performed using the Fine ${ }^{\mathrm{TM}} / \mathrm{Hexa}$ solver by Numeca Int. on a grid of 45,000 cells. The close up of grid layout is shown in Figure 10. A refined box is used to solve the shock accurately. The flow is modeled by the Euler equations and the mean free stream Mach number is set to $M=0.8$. The pressure coefficient is shown in Figure 11, indicating a clear shock starting at the upper surface at $x / c=0.7809$. The uncertainties are the camber $c$ and the relative thickness $t$ of the airfoil, both are assumed to have a truncated normal distribution, with the same parameters as the subsonic case.

Figure 12 shows the pressure coefficient at the surface of the airfoil. The mean is shown by the solid line, whereas the uncertainty bars indicate the $95 \%$ or $2 \sigma$ interval. Due to the presence of the shock the response surface contains a discontinuity. This causes the global polynomial approximation to oscillate and produce unphysical approximations, as can be observed by the large blue uncertainty bars near the shock location. This issue can be solved using a Multi-Element Probabilistic Collocation approach. ${ }^{12}$ Here a piecewise linear approximation through the collocation points is used to approximate the response surface without unphysical results. The results of the piecewise linear approximation are shown in Figure 12 by the red bars. 


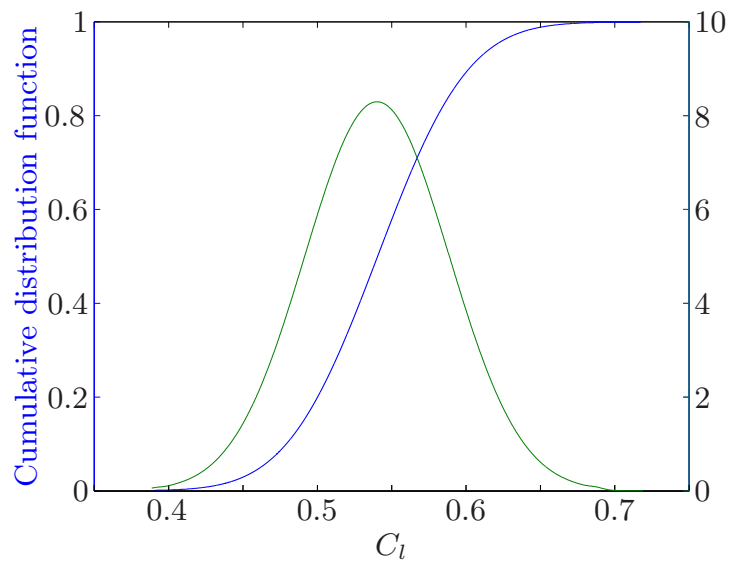

(a)

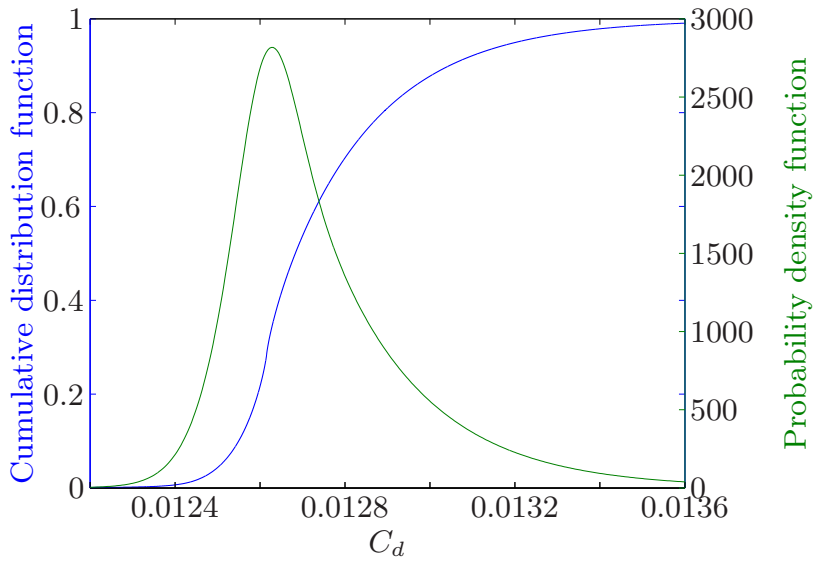

(b)

Figure 9. Probability distribution functions for $C_{l}$ and $C_{d}$ using a 2nd order Probabilistic Collocation approximation.

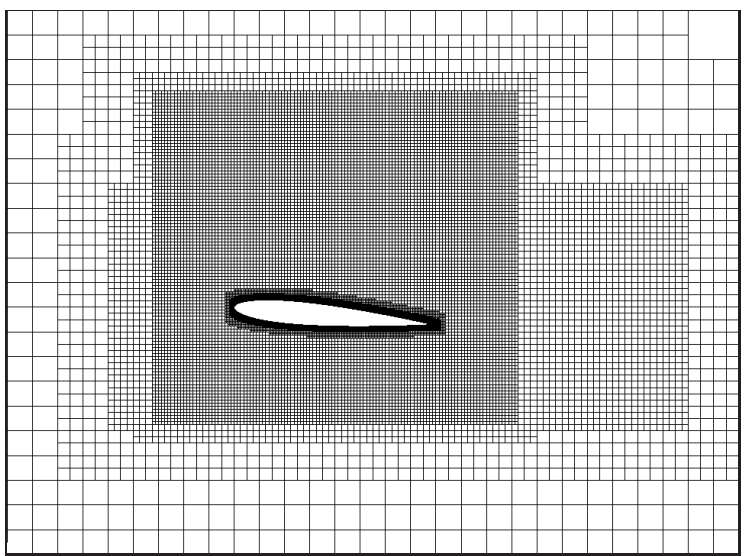

Figure 10. The computational mesh layout near the airfoil

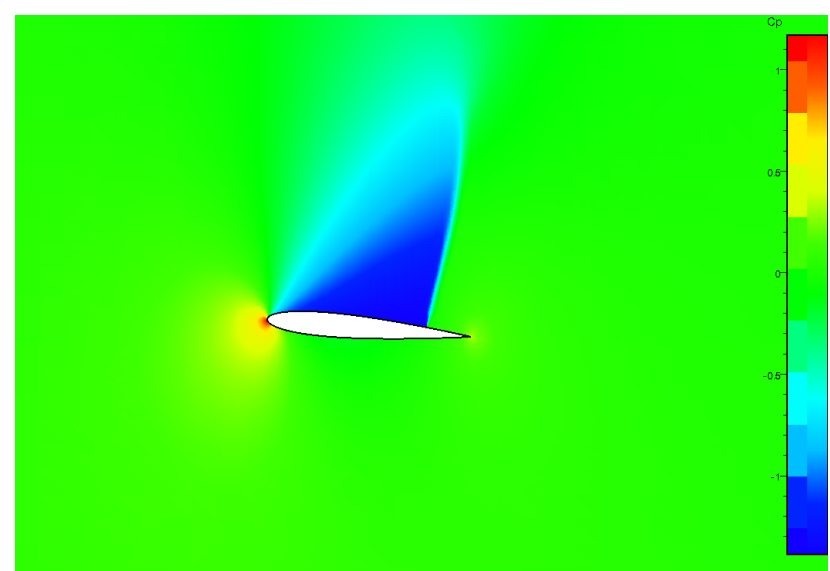

Figure 11. Pressure coefficient around the mean airfoil at $M=0.8$ 
The shock location it self is a output functional which is easily approximated using the Probabilistic Collocation method. The probability distribution functions are shown in Figure 13. The deterministic shock location is at $x_{\text {shock,det }}=0.7809$. The mean shock location based on the input uncertainties is $\mu_{x_{\text {shock }}}=0.7779$ with a standard deviation of $\sigma_{x_{\text {shock }}}=0.0288$ resulting in a coefficient of variation of $C V_{x_{\text {shock }}}=3.7 \%$. This variation is significant, the $95 \%$ area covers over $10 \%$ of the chord.

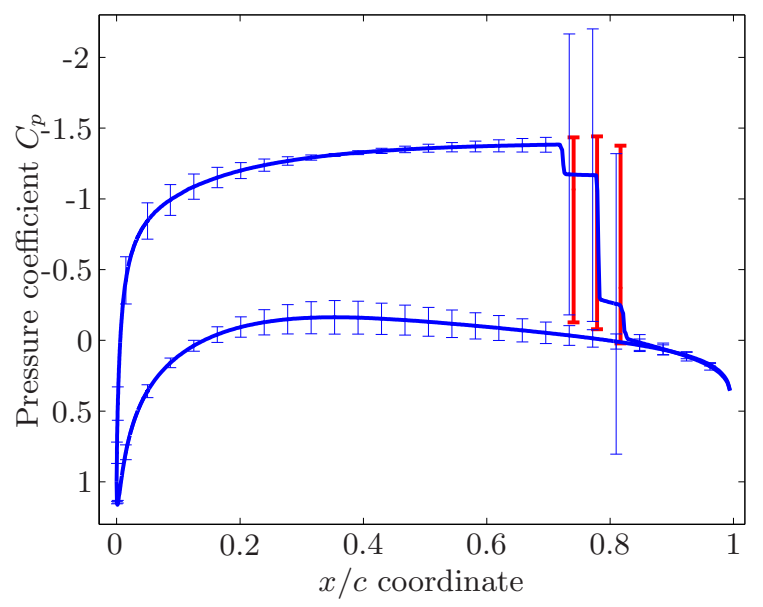

Figure 12. Pressure distribution at the surface of the airfoil. The mean is indicated with the solid blue line $(-)$, the uncertainty bars show the $2 \sigma$ region (containing 95\% of all values). The red bars $(-)$ show the piecewise linear approximation near the shock location.

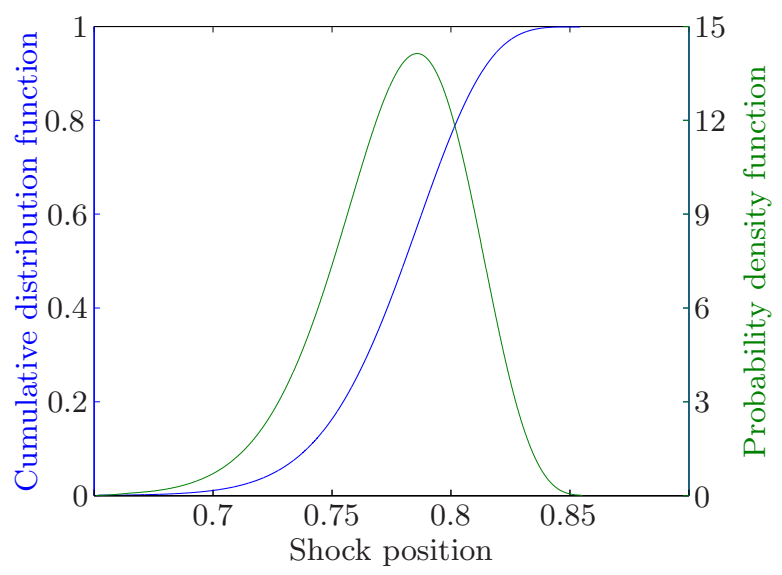

Figure 13. Probability distribution functions of the shock location using a 2nd order Probabilistic Collocation approximation.

\section{Conclusions}

The results in this paper show that effects of geometric uncertainties on the lift and drag coefficient of an airfoil are significant. The geometry was parameterized using the maximum camber, maximum camber location and thickness as parameters. The geometric uncertainty is treated as a parametric uncertainty, which is propagated using the Probabilistic Collocation method. The uncertainties are studied separately and combined. For the case of a NACA 4-digit series airfoil, the maximum camber has the largest influence on the solution, the input uncertainty can increase to a larger uncertainty in the solution. An uncertain maximum camber location results in only little uncertainty of the solution. The thickness is shown to be most important at higher angles of attack, where the turbulent separation from the trailing edge plays an important role. From the study of the combined effect of the three uncertain shape parameters it is shown that the uncertainty interval of the solution is significantly large. It is, therefore, important to include geometric uncertainties in the analysis of airfoil since they are inherently present in reality.

By describing the geometry of an airfoil with a few parameters, the uncertain geometry can be treated as parametric uncertainties. The Probabilistic Collocation method is used to propagate the parametric uncertainties. However, when the number of uncertain parameters increases the amount of computational work of the Probabilistic Collocation method will increase. The amount of uncertain parameters should, therefore, be limited depending of the order of approximation, which can be done by a suitable parameterization.

Two test cases are shown using a commercial CFD solver. The first case was turbulent flow around a NACA0012 airfoil at a Mach number of $M=0.3$ with uncertain camber and relative thickness. The uncertainties are propagated using a second order Probabilistic Collocation method with a commercial CFD solver to perform the required deterministic solves. The pressure distribution on the surface of the airfoil show that the uncertainties mainly affect the leading edge and the area around the maximum camber, between 20 and 60 percent of the chord. The effect on the lift and drag coefficient is significant. A spread in the solution of 190 lift- and 80 dragcounts are observed based on the input uncertainties. The second case was Euler flow around the same airfoil at a Mach number of $M=0.8$, again with uncertain camber and relative thickness. The shock location on the airfoil becomes uncertain and shows a coefficient of variation of $3.7 \%$. The area that contains $95 \%$ of all possible shock locations covers over $10 \%$ of the chord. 


\section{Acknowledgments}

The presented work is supported by the NODESIM-CFD project (Non-Deterministic Simulation for CFD based design methodologies); a collaborative project funded by the European Commission, Research Directorate-General in the $6^{\text {th }}$ Framework Programme, under contract AST5-CT-2006-030959.

\section{References}

${ }^{1}$ Ilinca, F., Hay, A., and Pelletier, D., "Shape Sensitivity Analysis of Unsteady Laminar Flow Past a Cylinder in Ground Proximity," Proceedings of the $36^{\text {th }}$ AIAA Fluid Dynamics Conference and Exhibit, AIAA paper 2006-3880, San Francisco, June 2006.

${ }^{2}$ Etienne, S., Hay, A., Garon, A., and Pelletier, D., "Shape Sensitivity Analysis of Fluid-Structure Interaction Problems," Proceedings of the $36^{\text {th }}$ AIAA Fluid Dynamics Conference and Exhibit, AIAA paper 2006-3217, San Francisco, June 2006.

${ }^{3}$ Gumbert, C. R., Newman, P. A., and Hou, G. J.-W., "Effect of Random Geometric Uncertainty on the Computational Desing of a 3-D Flexible Wing," Proceedings of the 20 $0^{\text {th }}$ AIAA Applied Aerodynamics Conference, AIAA paper 2002-2806, St. Louis, June 2002.

${ }^{4}$ Ghanem, R. G. and Spanos, P. D., Stochastic Finite Elements: A Spectral Approach, Dover, 1991.

${ }^{5}$ Babuška, I., Nobile, F., and Tempone, R., "A Stochastic Collocation Method for Elliptic Partial Differential Equations with Random Input Data," ICES report 05-48, 2005.

${ }^{6}$ Loeven, G. J. A., Witteveen, J. A. S., and Bijl, H., "Probabilistic Collocation: an efficient non-intrusive approach for arbitrarily distributed parametric uncertainties," Proceedings of the $45^{\text {th }}$ AIAA Aerospace Sciences Meeting and Exhibit, AIAA paper 2007-317, Reno, January 2007.

${ }^{7}$ Tatang, M. A., Pan, W., Prinn, R. G., and McRae, G. J., "An efficient method for parametric uncertainty analysis of numerical geophysical models," Journal of geophysical research, Vol. 102, No. D18, 1997, pp. 21925-21932.

${ }^{8}$ Golub, G. H. and Welsch, J. H., "Calculation of Gauss quadrature rules," Mathematics of Computation, Vol. 23, No. 106, 1969, pp. 221-230.

${ }^{9}$ Gautschi, W., "Orthogonal polynomials (in Matlab)," Journal of Computational and Applied Mathematics, Vol. 178, 2005, pp. 215-234.

${ }^{10}$ Gander, M. J. and Karp, A. H., "Stable computation of high order Gauss quadrature rules using discretization for measures in radiation transfer," Journal of Quantitative Spectroscopy Radiative Transfer, Vol. 68, 2001, pp. 213-223.

${ }^{11}$ Mathelin, L. and Hussaini, M. Y., "A Stochastic Collocation algorithm for uncertainty analysis," Tech. Report NASA/CR2003-212153, NASA Langley Research Center, 2003.

${ }^{12}$ Loeven, G. J. A., Sarkar, S., Witteveen, J. A. S., and Bijl, H., "Dynamic stall utter analysis with uncertainties using Multi-Element Probabilistic Collocation," Proceedings of the $9^{\text {th }}$ AIAA Non-Deterministic Approaches Conference, AIAA paper 2007-1964, Waikiki, April 2007.

${ }^{13} \mathrm{Xiu}, \mathrm{D}$. and Hesthaven, J., "High order collocation methods for differential equations with random inputs," SIAM J. Sci. Comput., Vol. 27, No. 3, 2005, pp. 1118-1139. 1980.

${ }^{14}$ Eppler, R. and Somers, D., "A Computer Program for the Design and Analysis of Low-Speed Airfoils," NASA TM 80210,

${ }^{15}$ Eppler, R., "Turbulent Airfoils for General Aviation," Journal of Aircraft, Vol. 15, No. 2, 1978.

${ }^{16}$ Hepperle, M., "JavaFOIL," 1996-2006, http://www.mh-aerotools.de/.

${ }^{17}$ Drela, M. and Giles, M. B., "Viscous-Inviscid Analysis of Transonic and Low Reynolds Number Airfoils," AIAA 861786-CP, 1986. 